\title{
Acute renal failure due to small cell neuroendocrine carcinoma of the left kidney: A case report
}

\author{
HAKAN BAHADIR HABERAL $^{1 *}$, ŞENOL TONYALI $^{1 *}$, DİLEK ERTOY BAYDAR $^{2^{*}}$ and CENK YÜCEL BİLEN ${ }^{1 *}$ \\ Departments of ${ }^{1}$ Urology and ${ }^{2}$ Pathology, Hacettepe University School of Medicine, Ankara 06230, Turkey
}

Received April 19, 2017; Accepted July 27, 2017

DOI: $10.3892 / \mathrm{ol} .2017 .6948$

\begin{abstract}
The majority of small cell carcinoma (SCC) cases originate from the lungs. SCC of the kidneys is rare. Among genitourinary tumors, renal cell carcinoma is the most common type of tumor to be associated with paraneoplastic syndromes. The majority of paraneoplastic syndromes disappear following nephrectomy in renal cell carcinoma cases. The present case involved the assessment of a female patient with SCC of the left kidney. The patient was diagnosed with acute renal failure and underwent a laparoscopic left radical nephrectomy. Surgical intervention removed the need for dialysis; the patient was under follow-up observation every week without any requirement for dialysis for 8 months and received adjuvant carboplatin-etoposide chemotherapy. Surgery is an option for dialysis-dependent patients with a renal tumor. The removal of the tumor burden may restore renal function and eliminate paraneoplastic syndromes.
\end{abstract}

\section{Introduction}

Small cell carcinoma (SCC) usually originates from the lungs and accounts for $\sim 12.95 \%$ of all lung cancer cases in the USA in 2002 (1). Extra-pulmonary SCC is commonly identified in the gastrointestinal tract, genitourinary organs, head or neck (2). While the prostate and bladder are the most common sites for SCC in the genitourinary tract, SCC of the kidneys is rare (3). For localized SCC, surgical treatment with adjuvant chemotherapy is the suggested option (2). Renal cell carcinoma is the most common type of genitourinary cancer associated with paraneoplastic syndrome (PNS), but the majority of PNS disappears following nephrectomy (4). The current case involved the assessment of a patient who underwent a left

Correspondence to: Dr Hakan Bahadır Haberal, Department of Urology, Hacettepe University School of Medicine, Gevher Nesibe Street, Ankara 06230, Turkey

E-mail: bahadirhaberal@gmail.com

*Contributed equally

Key words: dialysis dependence, increased glomerular filtration rate, kidney tumor, neuroendocrine tumor, small cell carcinoma radical nephrectomy due to SCC of the kidney. To the best of our knowledge, the present study is the first to describe a patient who regained renal function and did not depend on dialysis following a nephrectomy due to kidney SCC.

\section{Case report}

A 64-year-old female patient was admitted to Hacettepe University Hospital (Ankara, Turkey) in November 2016 with fatigue and chest pain. The patient presented had a medical history of asthma and hypertension. Laboratory examination revealed that the serum creatinine levels, blood urea nitrogen levels and the estimated glomerular filtration rate (eGFR) were $10.41 \mathrm{mg} / \mathrm{dl}$ (normal range, 0.5-1.3 mg/dl), $175.80 \mathrm{mg} / \mathrm{dl}$ (normal range, $8-21 \mathrm{mg} / \mathrm{dl}$ ) and 3 (normal, >60), respectively. Venous blood gas analysis revealed acidosis $(\mathrm{pH} 7.09$; normal range, 7.35-7.45). Due to these test results, the patient underwent a permanent dialysis catheter placement; the patient required dialysis by a hemodialysis machine for three consecutive days (2,3 and 4 h on day 1,2 and 3, respectively). The patient provided written informed consent for the publication of this case report.

To determine the cause of renal failure, abdominal ultrasonography (USG) was performed, which revealed a 95x84-mm solid mass in the left kidney, and hydronephrosis due to the pressure of the mass. To understand the structure of the mass and identify potential metastasis, abdominal magnetic resonance imaging (MRI) and thoracic computed tomography (CT) scans were performed. Abdominal MRI revealed a $93 \times 88-\mathrm{mm}$ solid lesion originating from the upper pole of the left kidney, but no evidence of metastasis (Figs. 1 and 2). The thoracic CT revealed no metastasis.

The patient underwent laparoscopic radical nephrectomy via a transperitoneal approach. On macroscopic examination, the resected kidney was determined to be $11 \times 11 \times 9 \mathrm{~cm}$. The cut surface of the organ revealed a solid mass (largest diameter, $10.5 \mathrm{~cm}$ ) that occupied the renal parenchyma almost entirely. The tumor filled out the collecting duct system, obliterated the pelvic cavity and invaded the perinephric and hilar fat tissue. The tumor reached Gerota's fascia with positive surgical margins. Following overnight fixation in $4 \%$ formaldehyde at room temperature, samples from the tumor and surrounding kidney were taken and subjected to routine paraffin embedding procedure. The paraffin-embedded samples (thickness, $5 \mu \mathrm{m}$ ) were initially stained with hematoxylin and eosin on Shandon Varistain Gemini (Shandon, Frankfurt, Germany) 
automated slide stainer (completed in $50 \mathrm{~min}$ at room temperature). Additional histochemical staining with Jones' methenamine silver method was performed to assess the non-neoplastic kidney morphology. For this, slides were kept in $0.5 \%$ working silver solution for $75 \mathrm{~min}$ at $70^{\circ} \mathrm{C}$. All light microscope evaluations were performed using Zeiss Axioscope. A1 optical microscope. Various magnifications (x4, x10, x20 or x40) were applied depending on the requirement. Tumor sections revealed neoplastic cells with narrow cytoplasm and small hyperchromatic irregular molding nuclei forming hypercellular sheets, nests, columns and rosette-like structures (Fig. 3). Large areas of necrosis and lymphovascular invasion were observed. Mitotic rate was high (>50/10 high power field) with abundant apoptotic figures.

An immunohistochemical panel was performed on the Leica-BOND-Max automated staining platform (Leica Microsystems, Wetzlar, Germany). Briefly, the tissue sections from the representative paraffin blocks that were cut at $4 \mu \mathrm{m}$ thickness onto charged slides were deparaffinized with Bond Dewax solution (Leica Microsystems; cat. no. AR9222) at $75^{\circ} \mathrm{C}$. Following the treatment through a $96 \%$ alcohol series and heat induced epitope retrieval with an EDTA-based buffer (Leica Biosystems, Newcastle Ltd., Newcastle Upon Tyne, UK; cat. no. AR9640) at $100^{\circ} \mathrm{C}$ for $10 \mathrm{~min}$, tissue sections were incubated for $25 \mathrm{~min}$ with the following primary antibodies at room temperature: Anti-cytokeratin 7 (Leica Microsystems; dilution, 1:100; cat. no. NCL-L-CK7-560), anti-synaptophysin (Leica Microsystems; dilution, 1:100; cat. no. NCL-L-SYNAP-299), anti-chromogranin (Invitrogen; Thermo Fisher Scientific, Inc.; dilution, 1:100; cat. no. MA5-13096), anti-thyroid transcription factor-1 (TTF-1) (Leica Microsystems; dilution, 1:200; cat. no. NCL-L-TTF-1), anti-Pax8 (Thermo Fisher Scientific, Inc.; dilution, 1:200; cat. no. MA1-117), anti-GATA binding protein-3 (Biocare Medical; dilution, 1:80; cat. no. CM 405 B) and Wilms Tumor-1 (Biocare Medical; dilution, 1:100; cat. no. CM 258 BK). Washing between the steps was accomplished by TBS (Leica Microsystems; cat. no. AR9590). Next, the Bond Polymer Refine Detection kit (Leica Biosystems, Newcastle Ltd.; cat. no. DS9800) was used for the detection of proteins. The kit included several reagents which were used sequentially: 3-4\% hydrogen peroxide to block endogenous peroxidase activity (13 $\mathrm{min}$ at room temperature), ready-to-use secondary antibody ( $9 \mathrm{~min}$ at room temperature), horseradish peroxidase- $\mathrm{IgG}$ polymer ( $9 \mathrm{~min}$ at room temperature), 3,3'-diaminobenzidine tetrahydrochloride (DAB) as a chromogen ( $7 \mathrm{~min}$ at room temperature) and hematoxylin for counterstaining (3 $\mathrm{min}$ at room temperature). Immunohistochemically stained slides were evaluated under the light microscope using various magnifications with $\mathrm{x} 4$, $\mathrm{x} 10, \mathrm{x} 20$ or $\mathrm{x} 40$ objectives. The tumor cells demonstrated diffuse and strong reactivity for cytokeratin 7, synaptophysin, chromogranin and TTF-1 (Fig. 4). No staining for pax 8, GATA-3 or WT-1 was observed. The diagnosis of pure small cell carcinoma (poorly differentiated neuroendocrine carcinoma) of kidney was rendered. No associating component of renal cell cancer or urothelial carcinoma was identified.

Non-neoplastic renal parenchyma outside the tumor reflected chronic alterations characterized by widespread tubular atrophy and interstitial fibrosis with scattered globally

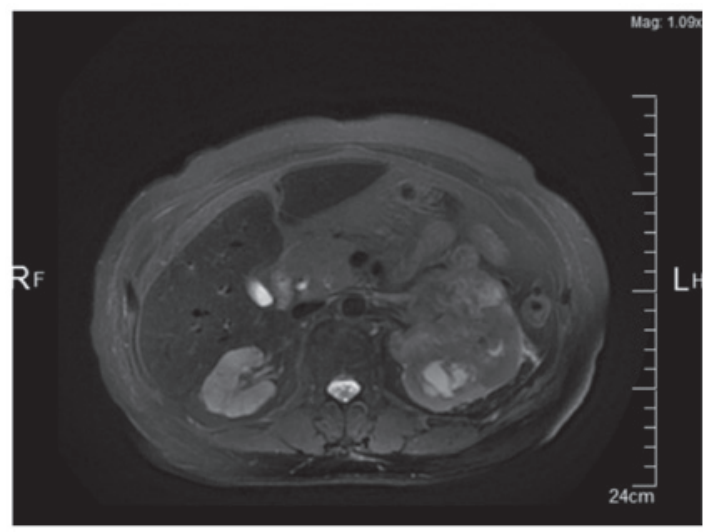

Figure 1. Magnetic resonance imaging of the tumor of the left kidney (transverse view; magnification, $\mathrm{x} 1.09)$.

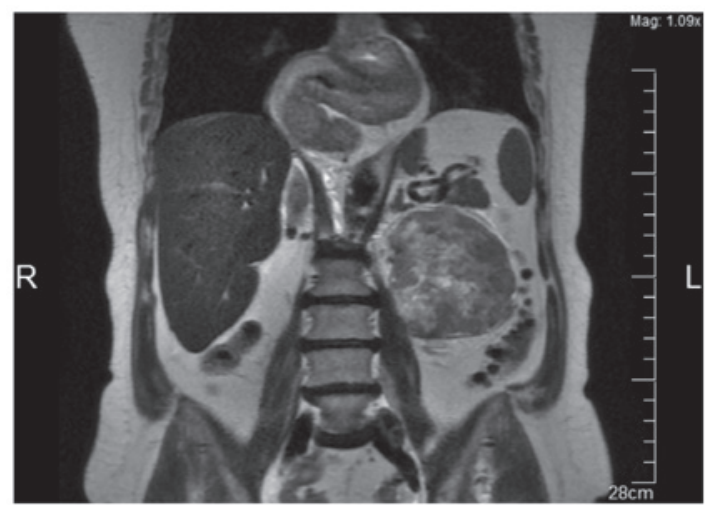

Figure 2. Magnetic resonance imaging of the tumor of the left kidney (coronal view; magnification, x1.09).

and segmentally sclerotic glomeruli (Fig. 5). There was a mild interstitial inflammatory infiltrate consisting of limited lymphocytes. No proliferative glomerular changes were identified. Immunofluorescence staining, performed on $2 \mu \mathrm{m}$ paraffin sections following antigen retrieval, as previously described (5), revealed no immunoglobulin (Ig) G, IgA, IgM, complement component $(\mathrm{C} 3, \mathrm{C} 4$ or $\mathrm{C} 1 \mathrm{q}) \kappa$ and $\lambda$ light chains deposition in the glomeruli, tubular basement membranes or blood vessels, which indicated absent expression. Considering the absence of proteinuria or hematuria in the patient, the observed chronic renal parenchymal changes were consistent with obstructive nephropathy induced by the tumor obliterating the pelvicalyceal system.

Control positron emission tomography/CT was performed and revealed no metastasis. The patient required dialysis 10 and 6 times prior to and following surgery, respectively. Subsequent to surgery, the patient received follow-up every week (for 8 months) without requiring dialysis or exhibiting evidence of disease. The patient's preoperative renal failure status was thought to be paraneoplastic due to the disappearance following nephrectomy of the primary tumor.

On the date of the final follow-up, serum creatinine levels, blood urea nitrogen levels and the eGFR were $3.97 \mathrm{mg} / \mathrm{dl}$ (normal range, $0.5-1.3 \mathrm{mg} / \mathrm{dl}$ ), $91.00 \mathrm{mg} / \mathrm{dl}$ (normal range, $8-21 \mathrm{mg} / \mathrm{dl}$ ) and 11 (normal, >60), respectively. Twelve-hour urine sample was collected. The collection of urine began in the morning. 


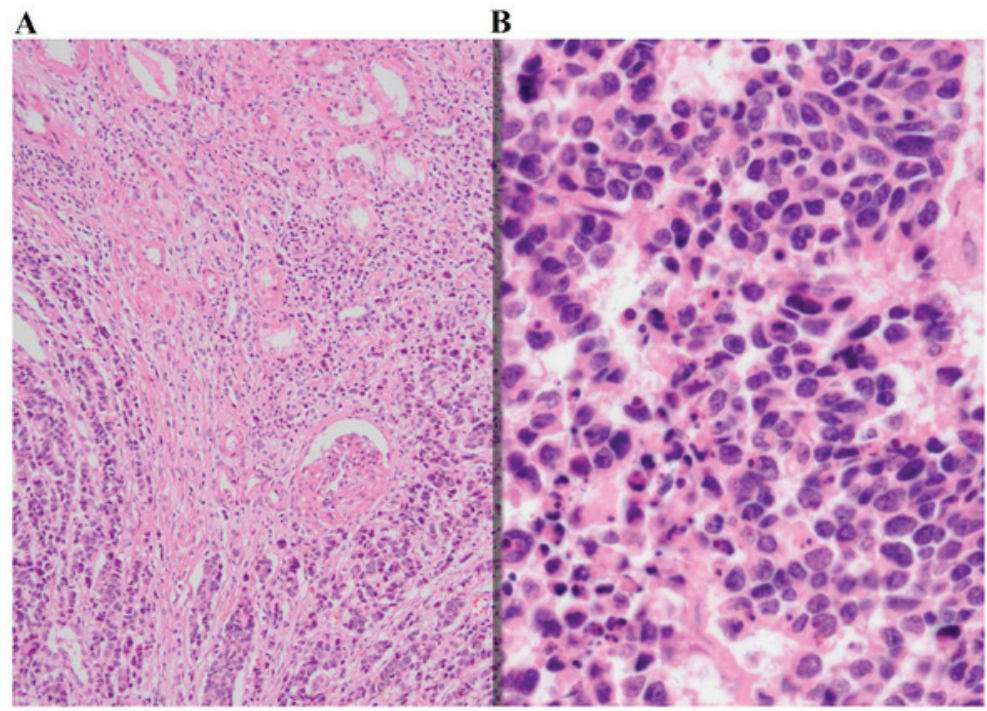

Figure 3. Tumor of the left kidney with typical features of small cell carcinoma. Analysis was performed using hematoxylin and eosin staining at magnification, (A) $x 100$ and (B) $\times 400$.

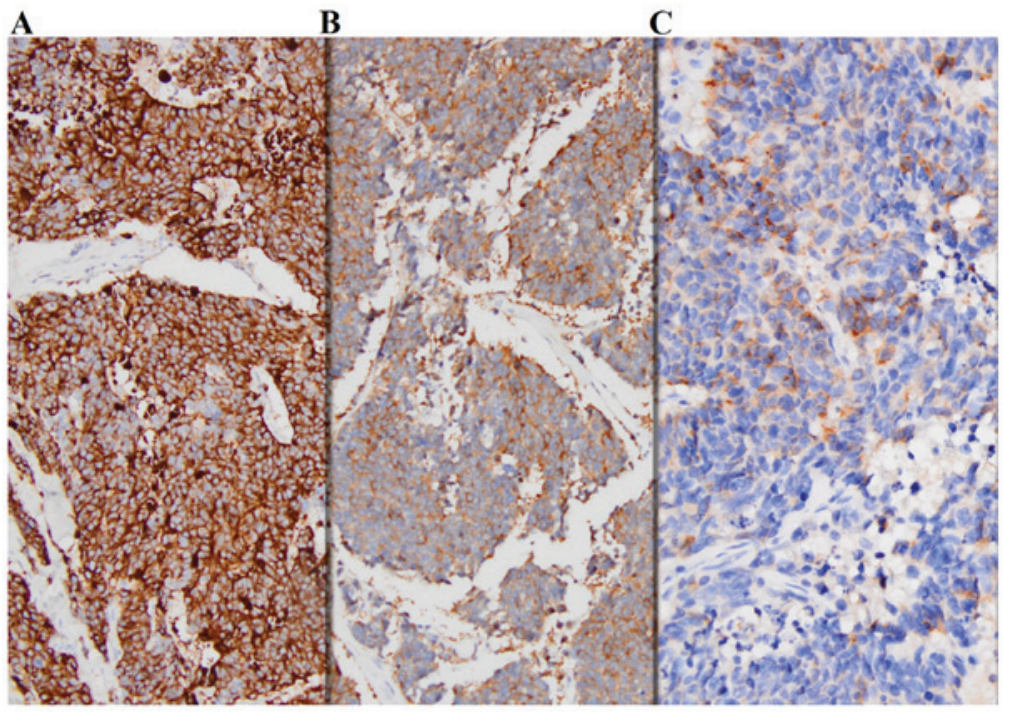

Figure 4. Immunohistochemical analysis of tumor-expressed components. Immunohistochemical analysis revealed that the tumor expressed (A) cytokeratin 7 (magnification, x100), (B) synaptophysin (magnification, x100) and (C) chromogranin (magnification, x200).

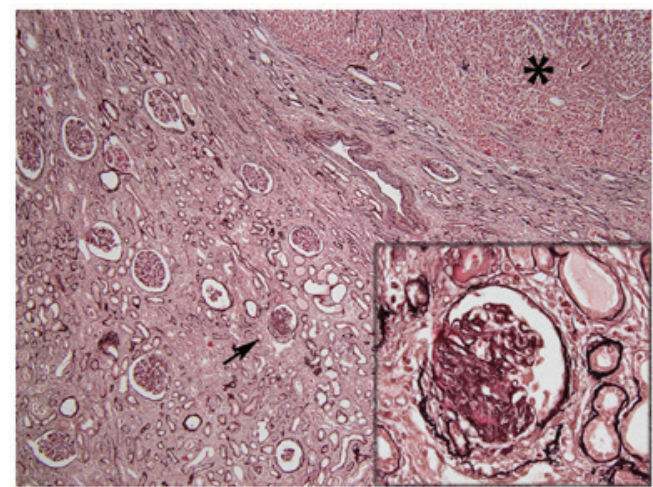

Figure 5. Light microscopy with Jones' methenamine silver stain highlighting chronic renal injury in non-neoplastic renal tissue, characterized by interstitial fibrosis, tubular atrophy and segmentally sclerosed glomerulus (denoted by an arrow and enlarged in the inset). Tumor tissue was observed in the upper right corner of the micrograph (denoted by an asterix). Magnification, $x 40$ inset magnification, $\mathrm{x} 400$.
Urine protein analysis $(12 \mathrm{~h}$ ) revealed $165.8 \mathrm{mg} / 12 \mathrm{~h}$ (normal, $150 \mathrm{mg} / 12 \mathrm{~h}$ ) proteinuria. The patient received adjuvant carboplatin-etoposide chemotherapy.

\section{Discussion}

Due to the rarity of neuroendocrine SCC of the kidneys, there are limited data associated with the natural course of and treatments for the disease. The existing data is based only on a few case reports and patient series.

Recommending treatments for extra-pulmonary SCC is challenging due to its multiple potential origins. However, a prior study suggested that patients should be grouped according to whether they exhibit localized or extended SCC. Chemotherapy is advised for patients with extended SCC, and surgical treatment with adjuvant chemotherapy is indicated for patients with localized SCC (2). The present study received 
carboplatin-etoposide adjuvant chemotherapy, as the literature suggested (2).

SCC of the kidneys was first described by Capella et al (6) in 1984. In 2003, Majhail et al (7) demonstrated that small cell neuroendocrine carcinoma of the kidney is $\sim 3.4$ times more common in females, as compared with renal cell carcinoma. The mean survival time of patients with small cell neuroendocrine carcinoma of a kidney is $<1$ year (7). Metastasis is observed in $\sim 60 \%$ of patients with small cell neuroendocrine carcinoma of the kidney, although there may be no sign of metastasis at the time of diagnosis (7). Lee et al (8) determined that the mean duration for recurrence is 4.8 months. Therefore, occult metastasis were considered a possible explanation for recurrence in these patients (8). A close follow-up after initial treatment is crucial for patients with small cell neuroendocrine carcinoma of the kidney due to the high risk of early local recurrence and distant metastasis (8). A cisplatin-based chemotherapy regimen has been demonstrated to improve survival $(7,8)$.

Following a radical nephrectomy, eGFR initially decreases but recovers during the long-term follow-up. Extended follow-up results for eGFR in patients who underwent radical nephrectomy were reported by Scosyrev et al (9) in 2014. While $85.7 \%$ of the patients underwent radical nephrectomy reached an eGFR of $<60$ during a 15 -year follow up, this rate had decreased to $58.7 \%$ at the time of the final follow-up (9).

All types of genitourinary cancer can cause paraneoplastic syndrome (PNS), but renal cell carcinoma is the most common type of tumor causally associated with PNS (4). Sacco et al (4) stated in 2009 that, among patients with renal cell carcinoma, $\sim 33 \%$ exhibit PNS. In patients with renal cell carcinoma, the majority of PNS cases disappear following nephrectomy (4). The majority of endocrinological PNS cases are associated with neuroendocrine tumors, particularly SCC (4). In the present study, acute kidney failure was hypothesized to be associated with PNS. Renal function improved following nephrectomy, which supported this hypothesis.

While hematological malignancies presenting with acute renal failure have previously been reported in the literature, to the best of our knowledge, this is the first study to assess a patient with a renal tumor who presented with acute renal failure (10).

To conclude, surgery may be considered as a treatment option in dialysis-dependent patients with renal tumors, and surgically eliminating the tumor burden may facilitate the recovery of patient eGFR.

\section{References}

1. Govindan R, Page N, Morgensztern D, Read W, Tierney R, Vlahiotis A, Spitznagel EL and Piccirillo J: Changing epidemiology of small-cell lung cancer in the United States over the last 30 years: Analysis of the surveillance, epidemiologic, and end results database. J Clin Oncol 24: 4539-4544, 2006.

2. Dakhil CS, Wick JA, Kumar AK, Satyan MT and Neupane P: Extrapulmonary small cell carcinoma: The University of Kansas experience and review of literature. Med Oncol 31: 187, 2014

3. Carranza OE, Castañón E, Abella LE, Zudaire ME, Castillo A, Arévalo E, Fusco JP, Zudaire JJ, Carias R, Cambeiro M, et al: Clinical management of small-cell carcinoma of the urinary tract: A 10-year single-center's experience. Clin Genitourin Cancer 11: 168-174, 2013.

4. Sacco E, Pinto F, Sasso F, Racioppi M, Gulino G, Volpe A and Bassi P: Paraneoplastic syndromes in patients with urological malignancies. Urol Int 83: 1-11, 2009.

5. Nasr SH, Galgano SJ, Markowitz GS, Stokes MB and D'Agati VD: Immunofluorescence on pronase-digested paraffin sections: A valuable salvage technique for renal biopsies. Kidney Int 70: 2148-2151, 2006

6. Capella C, Eusebi V and Rosai J: Primary oat cell carcinoma of the kidney. Am J Surg Pathol 8: 855-861, 1984.

7. Majhail NS, Elson P and Bukowski RM: Therapy and outcome of small cell carcinoma of the kidney: Report of two cases and a systematic review of the literature. Cancer 97: 1436-1441, 2003.

8. Lee SY, Hsu HH, Lin HY, Chen YC, Wong YC, Wang LJ, Ng KF, Chuang CK, Hung CC and Yang CW: Factors associated with the survival of patients with primary small cell carcinoma of the kidney. Int J Clin Oncol 18: 139-147, 2013.

9. Scosyrev E, Messing EM, Sylvester R, Campbell S and Van Poppel H: Renal function after nephron-sparing surgery versus radical nephrectomy: Results from EORTC randomized trial 30904. Eur Urol 65: 372-377, 2014.

10. Prakash J, Mandal AK, Vohra R, Wani IA, Hota JK, Raja R and Singh U: Renal disease is a prodrome of multiple myeloma: An analysis of 50 patients from eastern India. Ren Fail 31: 267-271, 2009. 